\title{
BOUNCING BALL MODES AND QUANTUM CHAOS
}

\author{
NICOLAS BURQ AND MACIEJ ZWORSKI
}

\begin{abstract}
Quantum ergodicity of classically chaotic systems has been studied extensively both theoretically and experimentally, in mathematics, and in physics. Despite this long tradition we are able to present a new rigorous result using only elementary calculus. In the case of the famous Bunimovich billiard table shown in Fig.1 we prove that the wave functions have to spread into any neighbourhood of the wings.
\end{abstract}

The quantum/classical correspondence is a puzzling issue that has been with us since the advent of quantum mechanics hundred years ago. Many aspects of it go back to the Newton/Huyghens debate over the wave vs. corpuscular theories of light.

On the surface of our existence we live in a world governed by laws of classical physics. That does not mean that we know precisely how fluids flow or solids move. They are described by highly non-linear rules which are hard to unravel mathematically. Even the simplest classical motion, that of a ball bouncing elastically from confining walls poses many unanswered questions - see http://wwr.dynamical systems.org/billiard/ for a fun introduction.

If we investigate deeper, or if we simply use any modern technical device, we come in contact with quantum mechanics. It is governed by a different set of rules which mix wave and matter. The simplest description of a wave comes from solving the Helmholtz equation:

$$
\left(-\Delta-\lambda^{2}\right) u=0, \quad \Delta=\partial_{x}^{2}+\partial_{y}^{2}, \quad(x, y) \in \Omega, \quad u \uparrow_{\partial \Omega}=0 .
$$

Here we put our wave inside of a two dimensional region $\Omega$. In classical wave mechanics the limit $\lambda \rightarrow \infty$ is described using geometrical optics where the waves propagate along straight lines reflecting in the boundary $\partial \Omega$. Roughly speaking, we expect something similar in the classical/quantum correspondence with the Helmholtz equation replaced by its quantum mechanical version, the Schrödinger equation. For many fascinating illustrations of this we refer to the web art gallery of Rick Heller: http://www.ericjhellergallery.com.

Many researchers on different aspects of semiclassical analysis have been interested in the the correspondence of solutions to the equation above and the classical geometry of balls bouncing from the walls of $\Omega$ : Bäcker, Cvitanović, Eckhardt, Gaspard, Heller, Sridhar, in physics, and Colin de Verdière, Melrose, Sjöstrand, Zelditch, in mathematics, to mention some (see [2], [3] for references to the physics literature, and [4, [5, [6] for mathematics).

Billiard tables for which the motion is chaotic are a particularly interesting model to study ${ }^{\dagger}$. One of the most famous is the Bunimovich billiard table shown in Fig.1. By adding two circular "wings" to a rectangular table the motion of a reflecting billiard ball becomes chaotic, or more precisely, hyperbolic, in the sense that changes in initial conditions lead to exponentially large changes in motion as time goes on.

\footnotetext{
†Of course one would not want to play billiards on a table like that, and a completely integrable rectangular one can pose enough of a challenge. While discussing billiards and the classical/quantum correspondence we cannot resist mentioning that Pyotr Kapitsa (Nobel Prize in Physics '78) was fond of saying that trying to detect the quantum nature of physical processes at room temperature was like trying to investigate the physical laws governing the collision of billiard balls on a table aboard a ship going through rough seas.
} 


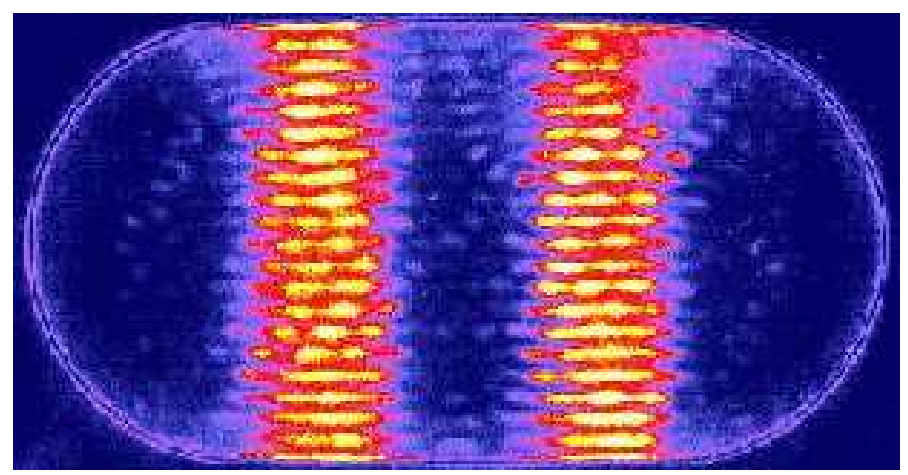

FiguRE 1. An experimental image of bouncing ball modes in a Bunimovich stadium cavity - see [1] and http://www.bath.ac.uk/ pyscmd/acoustics, With a certain amount imagination one can see our theorem in this picture.

As a model for studying quantum phenomena in chaotic systems this billiard table has become popular in experimental physics. A genuinely quantum example is shown in Fig.2 - it comes from the scanning tunnelling microscope work of Eigler, Crommie, and others 7 .

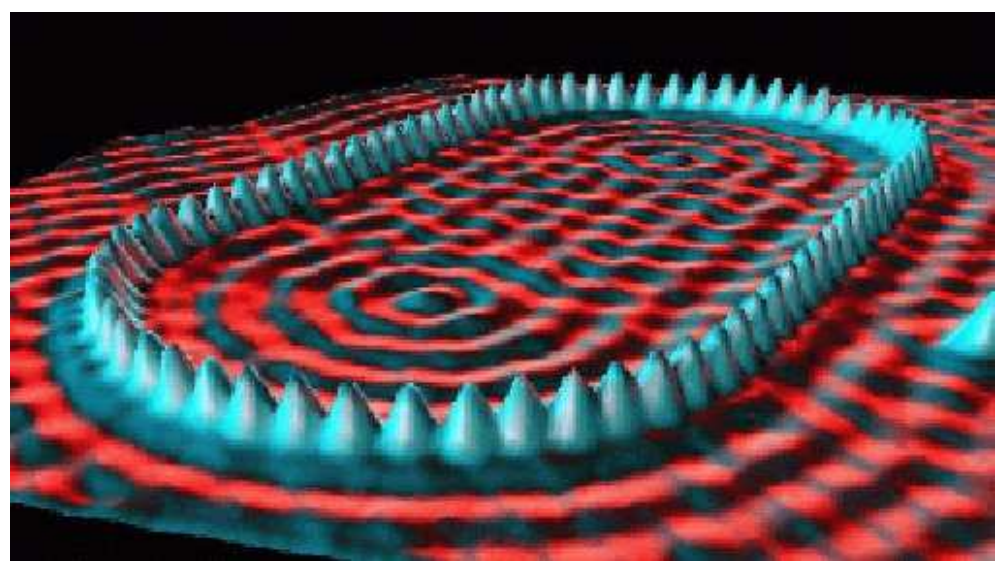

Figure 2. Quantum corral in the shape of the Bunimovich stadium. Courtesy of IBM® Research.

One question which is still mysterious to mathematicians and physicists alike is if the states of this system (that is, solutions of the equation above) can concentrate on the highly ustable closed orbits of the classical billiard. Quantum unique ergodicity states that there is no such concentration - see [8], [9], [6] and references given there. In the arithmetic case, that is for billiards given by arithmetic surfaces where the motion is given by the geodesic flow, spectacular advances have been recently achieved by Bourgain, Lindenstrauss [10, and Sarnak, while for the popular quantization of the Arnold cat map impressive results were produced by Bonechi, De Bièvre, Faure, and Nonnenmacher [11, and also by Kurlberg and Rudnick [12].

Here we describe an elementary but striking result in the billiard case. It follows from adapting the first author's earlier work in control theory. Although motivated by the more general aspects of [13] we give a simple self contained proof. 
For a state $u(x, y)$ what counts is its probability density, $|u(x, y)|^{2} d x d y$ - we assume here that $|u|^{2}$ is normalized to have integral 1 over $\Omega$. We say that it is bounded from below in a region if its integral over that region is bounded from below by a positive constant. With this terminology we have, roughly speaking,

Theorem. For any normalized state of the Bunimovich billiard table, the probability density in any neighbourhood of the wings of the table is bounded from below independently of the energy $\lambda^{2}$.

In particular, the result says that single bouncing ball orbits (that is orbits following an interval perpendicular to the horizontal straight boundaries) cannot produce localized waves. Our result allows concentration on the full invariant set of all vertical orbits over $R$ - that is consistent with the existing physical literature - both numerical and experimental - see [2] and [1]. In [13] we show a stronger result, namely that the neighbourhood of the wings can be replaced by any neighbourhood of the vertical intervals between the wings and the rectangular part. The proof of that predictable (to experts) improvement is however no longer elementary and is based on 14 .

The proof of theorem depends on the following unpublished result of the first author (see 13 for detailed references and background material):

Proposition. Let $\Delta=\partial_{x}^{2}+\partial_{y}^{2}$, be the Laplace operator on the rectangle $R=[0,1]_{x} \times[0, a]_{y}$. Then for any open $\omega \subset R$ of the form $\omega=\omega_{x} \times[0, a]_{y}$, there exists $C$ such that for any solutions of

$$
\left.\left(-\Delta-\lambda^{2}\right) u=f+\partial_{x} g \text { on } R, \quad u\right\rceil_{\partial R}=0
$$

with an arbitrary $\lambda \geq 0$ we have

$$
\int_{R}|u(x, y)|^{2} d x d y \leq C\left(\int_{R}\left(\left|f(x, y)^{2}\right|+|g(x, y)|^{2}\right) d x d y+\int_{\omega}|u(x, y)|^{2} d x d y\right) .
$$

Proof. We decompose $u$, and $f+\partial_{x} g$ in terms of the basis of $L^{2}([0, a])$ formed by the Dirichlet eigenfunctions $e_{k}(y)=\sqrt{2 / a} \sin (2 k \pi y / a)$,

$$
u(x, y)=\sum_{k} e_{k}(y) u_{k}(x), \quad f(x, y)+\partial_{x} g(x, y)=\sum_{k} e_{k}(y)\left(f_{k}(x)+\partial_{x} g_{k}(x)\right)
$$

we get for $u_{k}, f_{k}$ the equation

$$
\left(\partial_{x}^{2}+z\right) u_{k}=f_{k}+\partial_{x} g_{k}, \quad u_{k}(0)=u_{k}(1)=0, \quad z=\lambda^{2}-(2 k \pi / a)^{2} .
$$

It is now easy to see that

$$
\int_{0}^{1}\left|u_{k}(x)\right|^{2} d x \leq C\left(\int_{0}^{1}\left(\left|f_{k}(x)\right|^{2}+\left|g_{k}(x)\right|^{2}\right) d x+\int_{\omega_{x}}\left|u_{k}(x)\right|^{2} d x\right),
$$

where $C$ is independent of $\lambda_{1}$. In fact, let us first assume that $\omega_{x}=(0, \delta), \delta>0$, and $z=\lambda_{1}^{2}$, with $\operatorname{Im} \lambda_{1} \leq C$. We then choose $\chi \in \mathcal{C}_{\mathrm{c}}^{\infty}([0,1])$ identically zero near 0 and identically one on $[\delta / 2,1]$. Then

$$
\left(\partial_{x}^{2}+\lambda_{1}^{2}\right)\left(\chi u_{k}\right)=F_{k}, \quad F_{k}=\chi\left(f_{k}+\partial_{x} g_{k}\right)+2 \partial_{x} \chi \partial_{x} u_{k}+\partial_{x}^{2} \chi u_{k} .
$$

We can now use the explicit solution given by

$$
\chi(x) u_{k}(x)=\frac{1}{\lambda_{1}} \int_{0}^{x} \sin \left(\lambda_{1}(x-y)\right) F_{k}(y) d y .
$$


All the terms with $\partial_{x} g_{k}$ and $\partial_{x} u_{k}$ can be converted to $g_{k}$ and $u_{k}$ by integration by parts (with boundary terms 0 at both ends). Due to the $\lambda_{1}^{-1}$ factor that produces no loss and the estimate follows. The argument is symmetric under the $x \mapsto-x$ change, so we can place our control interval anywhere.

It remains to discuss the case $z \leq-C<0$. Then the estimate (2) follows from integration by parts (where now we do not need $\omega_{x}$ ):

$$
\begin{aligned}
& \int_{0}^{1}\left(f_{k}(x) \overline{u_{k}(x)}-g_{k}(x) \overline{\partial_{x} u_{k}(x)}\right) d x=\int_{0}^{1}\left(f_{k}(x)+\partial_{x} g_{k}(x)\right) \overline{u_{k}(x)} d x= \\
& \int_{0}^{1}\left(-\partial_{x}^{2}-z\right) u_{k}(x) \overline{u_{k}(x)} d x=\int_{0}^{1}\left(\left|\partial_{x} u_{k}(x)\right|^{2}+|z|\left|u_{k}(x)\right|^{2}\right) d x .
\end{aligned}
$$

By the Cauchy-Schwartz inequality, the left hand side is bounded from above by

$$
\left(\int_{0}^{1}\left(\left|f_{k}(x)\right|^{2}+\left|g_{k}(x)\right|^{2}\right) d x\right)^{\frac{1}{2}}\left(\int_{0}^{1}\left(\left|u_{k}(x)\right|^{2}+\left|\partial_{x} u_{k}(x)\right|^{2}\right) d x\right)^{\frac{1}{2}} .
$$

Since $|z|>C>0$, (2) follows from elementary inequalities (see [13 Lemma 4.1] for a general microlocal argument). We can now sum the estimate in $k$ to obtain the proposition.

We can now present a more precise version of the theorem. For a yet finer version we refer the reader to [13, Theorem $\left.3^{\prime}\right]$ and [13, Fig.5].

Theorem'. Consider $\Omega$ the Bunimovich stadium associated to a rectangle $R$. With the convention of Fig.1, let $R_{1}$ be any rectangle with the horizontal sides contained in the sides of $R$, strictly contained in $R$, and with $R \backslash R_{1}$ having two components.

There exists a constant $C$ depending only on $\Omega$ and $R_{1}$ such that for any solution of the equation

$$
\left(-\Delta-\lambda^{2}\right) v=f, \quad u \uparrow_{\partial \Omega}=0, \quad \lambda \geq 0,
$$

we have

$$
\int_{\Omega}|v(x, y)|^{2} d x d y \leq C\left(\int_{\Omega}|f(x, y)|^{2} d x d y+\int_{\Omega \backslash R_{1}}|v(x, y)|^{2} d x d y\right) .
$$

The "wings" of the billiard table in the original statement are given by $\Omega \backslash R_{1}$. We apply the second theorem with $f=0$ to obtain the first one.

Proof. Let us take $x, y$ as the coordinates on the stadium, so that $x$ is the horizontal direction, $y$ the vertical direction, and the internal rectangle is $[0,1]_{x} \times[0, a]_{y}$. Let us then consider $u$ and $f$ satisfying $\left(-\Delta-\lambda^{2}\right) u=f, u=0$ on the boundary of the stadium, and $\chi(x) \in \mathcal{C}_{\mathrm{c}}^{\infty}(0,1)$ equal to 1 on $[\varepsilon, 1-\varepsilon]$. Then $\chi(x) u(x, y)$ is solution of

$$
\left(-\Delta-\lambda^{2}\right) \chi u=\chi f+[\Delta, \chi] u \text { in } R
$$

with Dirichlet boundary conditions on $\partial R$. Since $[\Delta, \chi] u=2 \partial_{x}\left(\chi^{\prime} u\right)-\chi^{\prime \prime} u$ we can apply the proposition to obtain

$$
\int_{R}|\chi(x) u(x, y)|^{2} d x d y \leq C\left(\int_{R}|\chi(x) f(x, y)|^{2} d x d y+\int_{\omega_{\varepsilon}}|u(x, y)|^{2} d x d y\right)
$$

where $\omega_{\varepsilon}$ is a neighbourhood of the support of $\partial_{x} \chi$. Since we can choose it to be contained in $R \backslash R_{1}$, the theorem follows. 
We conclude by remarking that the same argument holds in the setting discussed recently in [15] and [6], since in the argument above the rectangle can be replaced by a torus.

Acknowledgments. The authors would like to thank the National Science Foundation for partial support under the grant DMS-0200732. They are also grateful to Arnd Bäcker, Stephan De Bièvre, Richard Melrose, and Steve Zelditch for helpful discussions and comments. They are also grateful to Paul Chinnery, Victor Humphrey, Don Eigler, and The IBM Corporation for the figures.

\section{REFERENCES}

[1] P.A. Chinnery and V.F. Humphrey. Experimental visualization of acoustic resonances within a stadiumshaped cavity. Physical Review E, 53:272-276, 1996.

[2] A. Bäcker, R. Schubert, and R. Stifter. On the number of bouncing ball modes in billiards. J. Phys. A: Math. Gen, 30:6783-6795, 1997.

[3] E.J. Heller. Quantum physics: Air juggling and other tricks. Nature, 412(33), 2001. News and Views.

[4] V.F. Lazutkin. KAM theory and semiclassical approximation to eigenfunctions, volume 24 of Ergebnisse der Mathematik und ihree Grenzgebiete. Springer Verlag, Berlin, 1993. With an addendum by A.I. Shnirelman.

[5] R.B. Melrose and J. Sjöstrand. Singularities of boundary value problems I \& II. Communications in Pure Applied Mathematics, 31 \& 35:593-617 \& 129-168, 1978 \& 1982.

[6] S. Zelditch. Quantum unique ergodicity. Preprint, 2003. http://www.arXiv.org/abs/math-ph/0301035

[7] M. F. Crommie, C. P. Lutz, and D. M Eigler. Confinement of electrons to quantum corrals on a metal surface. Science, 262(218), 1993.

[8] P. Sarnak. Arithmetic quantum chaos. In The Schur lectures (1992) (Tel Aviv), volume 8 of Israel Math. Conf. Proc., pages 183-236. Bar-Ilan Univ., Ramat Gan, 1995.

[9] A.I. Shnirelman. Ergodic properties of eigenfunctions. Uspekhi Mat. Nauk, 29:181-182, 1974.

[10] J. Bourgain and E. Lindenstrauss. Entropy of quantum limits. Comm. Math. Phys., 233(1):153-171, 2003.

[11] F. Faure, S. Nonnenmacher, and S. De Bievre. Scarred eigenstates for quantum cat maps of minimal periods. Preprint, 2003. http://www.arXiv.org/nlin.CD/0207060

[12] P. Kurlberg and Z. Rudnick. Value distribution for eigenfunctions of desymmetrized quantum maps. Internat. Math. Res. Notices, (18):985-1002, 2001.

[13] N. Burq and M. Zworski. Control in the presence of a black box. Preprint, 2003. http://www.arXiv.org/abs/math.AP/0304184

[14] C. Bardos, G. Lebeau, and J. Rauch. Sharp sufficient conditions for the observation, control and stabilization of waves from the boundary. S.I.A.M. Journal of Control and Optimization, 305:1024-1065, 1992.

[15] H. Donnelly. Quantum unique ergodicity. Proc. Amer. Math. Soc., 131:2945-2951, 2003.

Université Paris Sud, Mathématiques, Bât 425, 91405 Orsay Cedex

E-mail address: Nicolas.burq@math.u-psud.fr

Mathematics Department, University of California, Evans Hall, Berkeley, Ca 94720, USA

E-mail address: zworski@math.berkeley.edu 